\title{
World Journal of Surgery Becomes the Official Publication of the ERAS Society
}

\author{
Julie Ann Sosa ${ }^{1}$ Olle Ljungqvist ${ }^{2}$
}

Published online: 29 June 2018

(c) Société Internationale de Chirurgie 2018

It is with great excitement that we announce that the World Journal of Surgery has become the official journal for the Enhanced Recovery After Surgery (ERAS) Society. WJS continues to be the official journal of the International Society of Surgery (ISS). This new relationship with ERAS very much fits the mission of WJS, which is to promote innovation and discovery that helps to improve patient outcomes on the international stage.

The ERAS Society is a not-for-profit, multi-professional, and multidisciplinary international society with the mission to improve outcomes for patients undergoing surgery by education, scientific work, and implementation of evidence-based guidelines. The Society is engaged in publishing guidelines on various surgical procedures, as well as in promoting research collaborations. ERAS guidelines have been published for many operations, such as evidence-based modern care changes from overnight fasting to carbohydrate drinks $2 \mathrm{~h}$ before surgery, minimally invasive approaches instead of large incisions, management of fluids to seek balance rather than large volumes of intravenous fluids, avoidance of or early removal of drains and tubes, early mobilization, and serving of drinks and food the day of the operation. ERAS protocols have been associated with shorter length of hospital stay by more than $30 \%$ and similar reductions in complications, at the same time that readmissions and costs

Julie Ann Sosa

julie.sosa@ucsf.edu

Olle Ljungqvist

olle.ljungvist@oru.se

1 Department of Surgery, University of California at San Francisco-UCSF, San Francisco, CA, USA

2 Department of Surgery, Örebro University, Örebro, Sweden are reduced. The ERAS Society conducts structured implementation programs used in more than 20 countries. While ERAS started mainly with colorectal surgery, guidelines have been shown to improve outcomes in many surgical specialties.

Already, WJS has played a pivotal role in the dissemination of ERAS guidelines as well as research around implementation of the guidelines and their measurable impact on patient care. Impactful ERAS guidelines published by WJS include those describing optimal perioperative care following pancreaticoduodenectomy (2013), elective colonic surgery (2013), elective rectal/pelvic surgery (2013), bariatric surgery (2016), and elective liver surgery (2016) [1-5].

We look forward with excitement to our collaboration going forward and anticipate guidelines coming soon around perioperative care around esophagectomy. And we would like to thank Dr. John Hunter, former Editor in Chief of WJS, and Andrew Hill, President of the ISS, for facilitating this collaboration.

\section{References}

1. Lassen K, Coolsen MM, Slim K et al (2013) Guidelines for perioperative care for pancreaticodeuodenectomy: Enhanced Recovery after Surgery $\left(\right.$ ERAS $\left.^{\circledR}\right)$ Society recommendations. World J Surg 37(2):240-258. https://doi.org/10.1007/s00268-0121771-1

2. Gustafsson UO, Scott MJ, Schwenk W et al (2013) Guidelines for perioperative care in elective colonic surgery: Enhanced Recovery After Surgery $\left(\mathrm{ERAS}^{\circledR}\right)$ Society recommendations. World J Surg 37(2):259-284. https://doi.org/10.1016/j.clnu.2012.08.013

3. Nygren J, Thacker J, Carli F et al (2013) Guidelines for perioperative care in elective rectal/pelvic surgery: Enhanced Recovery After Surgery $\left(\right.$ ERAS $\left.^{\circledR}\right)$ Society recommendations. 
World J Surg 37(2):285-305. https://doi.org/10.1007/s00268-012$1787-6$

4. Thorell A, MacCormick AD, Awad S et al (2016) Guidelines for perioperative care in bariatric surgery: Enhanced Recovery After Surgery (ERAS) Society recommendations. World J Surg 40(9):2065-2083. https://doi.org/10.1007/s00268-016-3492-3
5. Melloul E, Hübner M, Scott M et al (2016) Guidelines for perioperative care for liver surgery: Enhanced Recovery After Surgery (ERAS) Society recommendations. World J Surg 40(10):2425-2440. https://doi.org/10.1007/s00268-016-3700-1 